\title{
Erzählen Sie Geschichten
}

Geschichten von Menschen, die Mathematik gemacht haben, erzähle ich gerne und lasse ungern eine Gelegenheit dazu aus. Geschichten gehören einfach in den Mathematikunterricht. Vielleicht ist die folgende Liste für Sie eine Anregung, Geschichten, Biografien und Anekdoten nicht zu kurz kommen zu lassen. Man könnte erzählen von

- Platon, den platonischen Körpern und den platonischen Ideen

- Euklid und seinem Buch

- Scipione del Ferro, Tartaglia, Cardano und der Gl. 3. Grades

- Newton, Leibniz, der Geschichte der Analysis und dem Infinitesimalen

- Ruffini, Abel, Galois,

- der Gl. 5. Grades, dem Anfang der modernen Mathematik und dem Abelpreis

- Euler, der e-Funktion und den Brücken in Königsberg

- Kant, Raum und Zeit und der Raumzeit bei Einstein

- Gauß, dem 10-DM-Schein und der Glockenkurve

- Hilbert und vom Ende der Mathematik in Göttingen nach 1933

- Andrew Wiles und dem großen Satz von Fermat

- Nicolas Bourbaki

- Emmy Noether und Sonja Kovalevskaja

- Gert Faltings, Maryam Mirzakhani, Peter Scholze und der Fieldsmedaille

- und von vielen, vielen anderen.

Und wo findet man die Biografien dieser Mathematikerinnen und Mathematiker? Suchen Sie z. B. in Wikipedia oder auch in Fröba und Wassermann (2013). 